\title{
Natural products at the Hans Knöll Institute
}

\begin{abstract}
Understanding the creation, induction and function of natural products that are important for microbial communication are central aims for scientists at the Leibniz Institute for Natural Product Research and Infection Biology - Hans Knöll Institute.
\end{abstract}

The history of the Hans Knöll Institute (HKI, http://www.hki-jena.de) begins in 1950 with Hans Knöll, a key player in developing production processes for penicillin and the director of the original Institute housed on the current site in Jena, Germany. Renamed in Knöll's honor in 1991 during a substantial reorganization, the HKI has stayed true to his memory, continuing investigations into diverse bacterial and fungal species and trying to learn more about the small molecules those species produce. In 1999, success on this front was recognized by the scientific council of Germany, the Wissenschaftsrat, resulting in the HKI's introduction to the Leibniz Association in 2003.

The Leibniz Institutes are unique among related research organizations (such as the Max Planck, Helmholtz, and Fraunhofer organizations) in that the Leibniz Association (http://www.wgl.de) selects pre-existing institutes to join the organization. The 84 Leibniz Institutes have further found a unique niche within the German research environment; they are neither solely focused on basic research like the Max Planck Institutes nor as industrially minded as the Fraunhofer Institutes. Rather, they are charged with the intermediate goal of 'theoria cum praxi,' or combining basic research with practical utility. The connection with the Leibniz Association is also not necessarily permanent: the selected institutes undergo periodic evaluations by the Association to insure that the research remains of the highest quality and that the research topic remains of central importance.

Current research at the HKI focuses on deciphering the biosynthesis of natural products and the cues that induce these processes, as well as the biological function of the compounds, all within the realm of interspecies communication in infectious diseases. The departments cover all aspects of this central topic, ranging from infection biology (including innate immunity, under the direction of Peter Zipfel) to the elucidation and engineering of natural product pathways (under the direction of Christian Hertweck) to the recent addition of a department interested in determining mechanisms of microbial pathogenicity (under the direction of Bernhard Hube). As Axel Brakhage, director of the HKI and of the Molecular and Applied Microbiology Department puts it, "we identified this research area and then gathered scientists with complimentary expertise; this interdisciplinarity really pulls everything together." While the entire Institute seems as collaborative as the microbial communities it studies, two divisions are particularly important to this interconnectivity: the Bio Pilot Plant (BPP, or Biotechnikum) and the Internal Product Line (IPL, or Durchgehende Bearbeitungslinie).

The BPP, directed by Uwe Horn, is a processing facility for the growth of bacteria and fungi in volumes up to 3,000 l, which allows production of significant quantities of a given natural product for further testing. In addition, the group has developed special machinery for screening a large variety of growth conditions at the nanoliter scale to determine suitable procedures for handling otherwise unculturable species. Horn notes that "it is very unique to have a pilot plant that can work with significantly different organisms.” In concert with the BPP, the HKI houses a collection of more than 36,000 bacterial and fungal isolates, which are now accessible to external scientists. Even with this extensive collection of microorganisms, Brakhage notes, "scientists estimate now that there are more than
1 million species of bacteria alone. It's incredible. What we have is only the tip of the iceberg." In describing his department's research, Hube says that knowledge of the microbial complexity of the natural world has inspired him; in particular, he hopes to understand the biochemical basis for why closely related microorganisms are pathogenic or not.

The focus of the IPL, which is coordinated by Ute Möllmann, is on the natural compounds produced by these diverse species, with each department contributing time and resources to the overall process. For example, Isabel Sattler's group within the Biomolecular Chemistry Department focuses on the initial identification of the compounds. The molecules are then subjected to assays that provide preliminary information on their biological function before they are added to the Institute's collection of more than 13,000 natural products and derivatives thereof. Having an understanding of the possible role of the compounds allows the HKI scientists to pursue further investigations, collaborations or

"I think we're only at
the very beginning of
understanding how things
work and how everything
is interconnected."
- Christian Hertweck

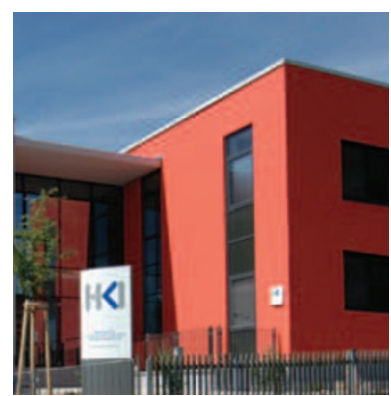

industrial contacts; Sattler laughingly describes her part in the process by saying, "I'm like a broker of natural products." For those compounds that aren't readily produced by the microorganisms in sufficient quantities (or at all, owing to lack of the requisite external cues), Hertweck's group is developing new strategies to find the compounds and to identify general biosynthetic principles.

The Institute's dedication to praxi is evident in several ways. So far nine companies have started as direct outgrowths of research at the HKI, and scientists (in particular, Alexander Tretjakov) within the Department of Cell and Molecular Biology created the 'Speedcycler', a variation of PCR that decreases experiment time from $3 \mathrm{~h}$ to $10 \mathrm{~min}$. This innovative atmosphere is not driven by an industrial bottom line, but rather the need for technology to keep pace with scientific curiosity. As Frank Hänel, another scientist in the same department, puts it, "when you want to answer a question, and there's not a way to do it, you simply develop new ways."

Jena itself is an excellent match for this intellectual outpouring. The city houses several other scientific companies and institutes, including the Friedrich Schiller University, where each department head from the HKI holds a faculty position, and the Max Planck Institute for Chemical Ecology, a collaborator in joint educational grants. As a result of this bustling scientific culture, Jena was recently awarded Germany's "City of Science" title for 2008. Just as Jena has been recognized for its high density of high-quality science, the HKI aims to capitalize on its unique organization to further our understanding of infection and how infection can be overcome. Indeed, in the quest for developing ideas about how fungi receive and respond to chemical messages, Hertweck says, "I think we're only at the very beginning of understanding how things work and how everything is interconnected."

Catherine Goodman, Jena, Germany 